\title{
On the orbital period distribution of cataclysmic variables
}

\author{
Coel Hellier and T. Naylor \\ Department of Physics, Keele University, Keele, Staffordshire, ST5 5BG
}

\begin{abstract}
Using the latest compilation of cataclysmic variable orbital periods by Ritter \& Kolb we argue against Verbunt's conclusion that the period gap is not significant for novalike variables. We also discuss the relation of the VY Scl stars to the dwarf novae.
\end{abstract}

Key words: accretion, accretion discs - novae, cataclysmic variables - binaries: close.

\section{INTRODUCTION}

Two fundamental characteristics of a cataclysmic variable $(\mathrm{CV})$ are the orbital period, $P$, typically between $80 \mathrm{~m}$ and $9 \mathrm{~h}$, and the behaviour of the optical lightcurve, showing either the recurrent 2-5 mag outbursts of a dwarf-nova (DN), or the steady lightcurve of a nova-like variable (NL). DN are thought to be low accretion rate $(\dot{M})$ systems with accretion discs cool enough to undergo hydrogen ionization instabilities, whereas NLs are always too hot for this to occur (see Warner 1995 for a comprehensive review of the whole field). The NLs predominantly have $P>3 \mathrm{~h}$, implying a high $\dot{M}$ and thus high angular momentum loss from the binary in this range. Systems with $P<2 \mathrm{~h}$ are predominantly DN, implying a lower angular momentum loss from gravitational radiation alone. Fewer systems occur in the 'period gap' between 2 and $3 \mathrm{~h}$, a possible consequence of the mechanism for additional braking switching off at $\sim 3 \mathrm{~h}$.

However, Verbunt (1997) has concluded that the period gap is not significant for NLs, and thus queried whether the additional braking mechanism (usually suggested to be braking by the magnetic field of the secondary) is required. In this paper we re-examine the issue using Ritter \& Kolb's (1998) more recent list of CVs with known orbital period. Further, we address the nature of VY Scl stars, which are currently poorly understood (e.g. Livio \& Pringle 1994; Wu, Wickramasinghe \& Warner 1995; Verbunt 1997).

\section{THE SAMPLE}

The philosophy of this paper is that we can use the outburst properties recorded by Ritter \& Kolb (1998) as an indicator of $\dot{M}$ (c.f. Shafter 1992). Thus we created a sample of high $\dot{M}$ systems into which we placed the NLs. Then, in a divergence from the method of Verbunt (1997), we removed all the NLs listed by Ritter \& Kolb (1998) as certain AM Her stars, DQ Her stars, or intermediate polars. This is because AM Hers don't possess discs and thus the presence or absence of outbursts is no longer an indicator of $\dot{M}$ (the use of the term 'nova-like' for AM Her stars is more historical than physical). Similarly, while intermediate polars mostly have discs, the magnetic field also affects the outburst behaviour (e.g. Warner 1996; Hellier, Mukai \& Beardmore 1997). We then created versions of the 'high $\dot{M}$ ' sample both including and excluding old novae, in case the nova eruption leaves the $\mathrm{CV}$ with an $\dot{M}$ temporarily atypical of its $P$. Lastly, since we wanted to investigate the status of VY Scl stars we removed them from the NL sample and placed them into a separate 'VY' sample. We refer to the high $\dot{M}$ sample as 'nNL' for 'normal NLs' (i.e. no magnetics or VY Scl stars) or as ' $\mathrm{nNL}+\mathrm{N}$ ' when it includes the old novae (recurrent novae were excluded from both samples).

A further reason for omitting old novae and magnetic systems is the potential bias due to selection effects: nova explosions draw attention to the underlying $\mathrm{CV}$, and the majority of magnetic systems are first seen in X-rays, whereas non-magnetic CVs are nearly all discovered optically (the variability of DN also make them more obvious than NLs, but as long as this affects all orbital periods equally it won't affect our analysis).

The Z Cam stars are hybrids showing periods of DN outbursts and periods of 'standstill' in which they act as NLs. The accepted explanation (e.g. Warner 1995) is that their $\dot{M}$ is finely poised at the boundary between NL and DN behaviour, so that a minor excursion moves them from one class to the other. We place these stars in a medium $\dot{M}$ sample called 'ZC'. Our low- $\dot{M}$ sample, essentially the DN, contains all systems that can never sustain an excursion to the hot side of the disc instability. We call these ' $\mathrm{nDN}$ ' for 'normal DN', to denote our exclusion of the Z Cams.

\section{IS THERE A PERIOD GAP?}

In Fig. 1 we plot the cumulative period distribution for our low- $\dot{M}$ sample (nDN) against the sample of all medium and high $\dot{M}$ systems (i.e. nNL+ZC+VY). There are clear breaks at $2.1 \mathrm{~h}($ in $\mathrm{nDN})$ and $\sim 3 \mathrm{~h}($ in $\mathrm{nNL}+\mathrm{ZC}+\mathrm{VY})$ and a deficit of systems in-between.

In case the reader is suspicious of our concocted high + medium- $\dot{M}$ sample, we first test the significance of the 


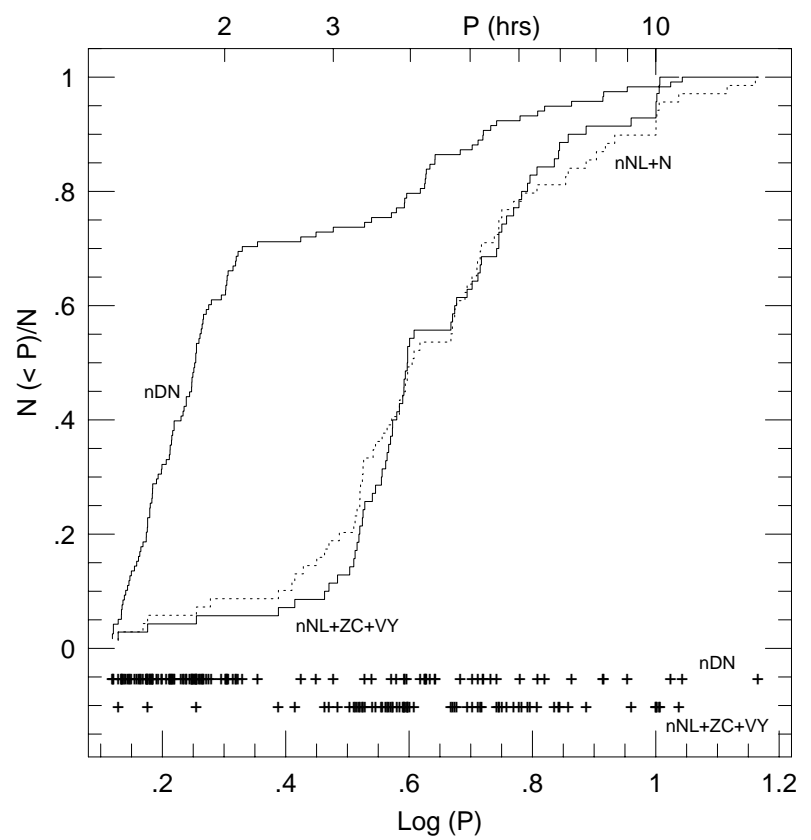

Figure 1. The cumulative orbital period distribution of low $\dot{M}$ CVs (nDN) compared with that of medium and high $\dot{M}$ CVs $(\mathrm{nNL}+\mathrm{ZC}+\mathrm{VY})$. The dashed line is the $\mathrm{nNL}+\mathrm{N}$ sample.

upper edge of the period gap with the purer nNL sample. A one-sided Kolmogorov-Smirnov (K-S) test shows that the distribution of nNL between 6 and $3 \mathrm{~h}$ is compatible with arising from a parent distribution of constant probability in $\log P$ at 67 per cent confidence. When testing against a constant distribution from $6 \mathrm{~h}$ to $2.1 \mathrm{~h}$ (where the DN turn on) the probability drops to 2 per cent, and when testing down to the $80-\mathrm{m}$ period minimum the probability drops to $10^{-4}$. For the other samples the drops in probability against a constant distribution in $\log P$ when the range is extended from $7-3 \mathrm{~h}$ to $7-2.1 \mathrm{~h}$ are: $\mathrm{nNL}+\mathrm{N}, 53$ to 1 per cent; $\mathrm{nDN}$, 43 to 2 per cent; and for $\mathrm{nDN}+\mathrm{ZC}, 41$ to 0.2 per cent. Thus all samples, including samples which are independent, show a significant (>95 per cent) reduction in the number of CVs below $\sim 3 \mathrm{~h}$. This conclusion differs from that of Verbunt (1997) because he included magnetic systems amongst the NLs. The evolution of magnetic systems may well be different from that of non-magnetics (e.g. Wickramasinghe \& Wu 1994), and using a two-sided K-S test for the current sample of systems with $P<5$ h gives only a 15 per cent probability that the distribution of AM Her stars and that of all non-magnetic CVs arise from the same parent population.

Turning now to shorter periods, the NLs are too few to investigate the significance of the break at $2 \mathrm{~h}$, so we do this with the low and medium $\dot{M}$ systems. Both nDN and $\mathrm{nDN}+\mathrm{ZC}$ are consistent with a constant distribution in $\log P$ between $80 \mathrm{~m}$ and $2.1 \mathrm{~h}$ at 32 per cent probability, but have only a $10^{-6}$ probability of constant distribution up to $3 \mathrm{~h}$. Thus the numbers of DN decline at $2 \mathrm{~h}$, significantly before the rise in NL numbers at $3 \mathrm{~h}$.

Verbunt (1997) has noted that the gap is most significant when using different samples above and below the gap (NL and DN respectively), raising the possibility of awkward selection effects. However, both the $\mathrm{nDN}$ and $\mathrm{nDN}+\mathrm{ZC}$ samples, which are homogeneous observational samples of all systems reliably showing DN outbursts, show both the

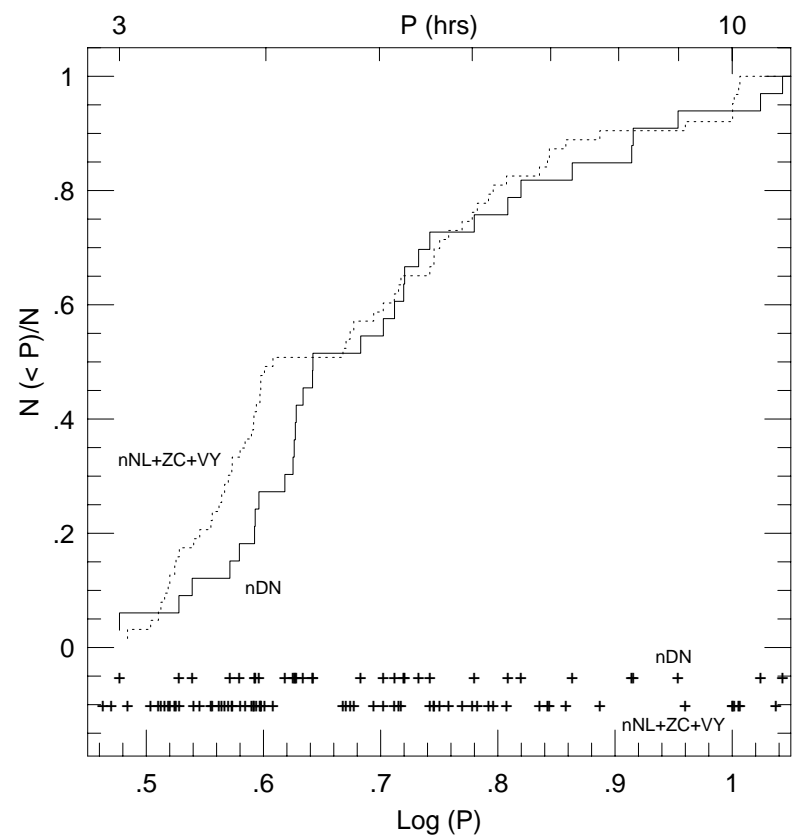

Figure 2. The distribution of low $\dot{M} \mathrm{CVs}(\mathrm{nDN})$ above the gap, compared with that of higher $\dot{M} \mathrm{CVs}(\mathrm{nNL}+\mathrm{ZC}+\mathrm{VY})$.

$2 \mathrm{~h}$ break and the $3 \mathrm{~h}$ break by themselves (>98 per cent confidence). Thus, regardless of the merits of the magnetic braking theory, the period gap is an observational fact. Since the orbital cycle is not used in the initial discovery of most $\mathrm{CVs}$, selection effects could only operate if periods of certain lengths were much harder to detect in known CVs. While lengths comparable to or longer than a night's observing $(P \gtrsim 6 \mathrm{~h})$ may indeed be harder to detect, we can think of no reason why periods of $2.5 \mathrm{~h}$ would be less obvious than those of $1.5 \mathrm{~h}$ or $3.5 \mathrm{~h}$.

\section{THE NATURE OF VY SCL STARS}

Considering only the stars above the gap $(P>3 \mathrm{~h})$, the samples $\mathrm{nNL}, \mathrm{nDN}$ and $\mathrm{ZC}$ are all compatible with arising from the same parent distribution (at 55 per cent probability for $\mathrm{nNL}$ and $\mathrm{nDN}$, and at 67 and 87 per cent probabilities for ZCs against $n N L s$ and $\mathrm{nDN}$ respectively). VYs occur preferentially just above the period gap, with all but two systems (whose periods are uncertain) in the range $3<P<4 \mathrm{~h}$.

Testing the low $\dot{M}$ sample (nDN) against higher $\dot{M}$ systems $(\mathrm{nNL}+\mathrm{ZC}+\mathrm{VY})$, provides evidence for a deficit of DN in the 3-4 h range (Fig. 2). Shafter (1992) reported this at 98 per cent significance. However, because of the new discoveries included in our sample, a two-sided K-S test now gives a 17 per cent probability that they come from the same parent distribution. Hence, while we need an explanation for the occurrence of VYs in this range, the lack of DN might be real, or might simply be a chance occurrence.

One suggested explanation is that DN turn into VYs in the 3-4 h range (see Livio \& Pringle 1993 and Verbunt 1997). We now argue that this is unlikely. The VYs have long stretches acting as NLs, and have absolute magnitudes typical of NLs rather than DN (e.g. Warner 1995). Thus to turn a DN into a VY we need to increase $\dot{M}$. However, we think we know what happens to a DN as $\dot{M}$ increases: it 


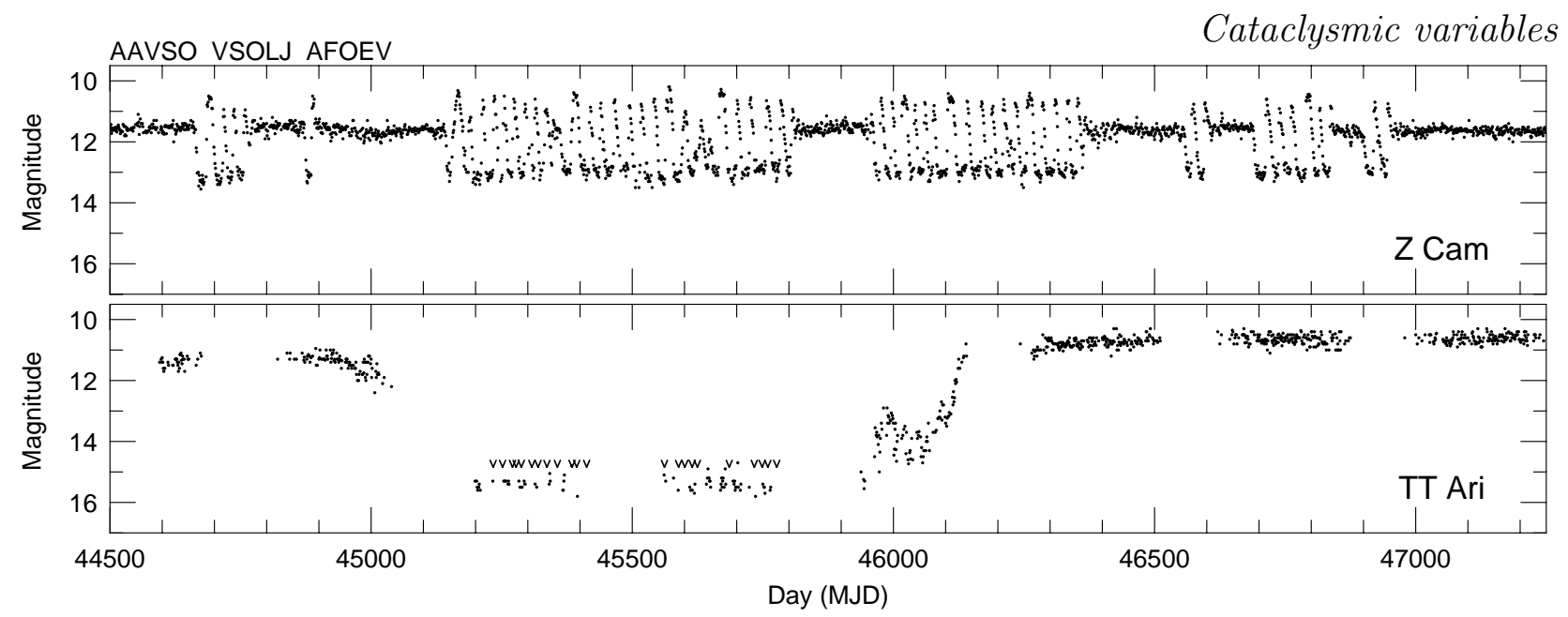

Figure 3. Longterm lightcurves of Z Cam and TT Ari, a typical VY Scl star. The relatively high $\dot{M}$ in Z Cam ensures frequent, regular outbursts when not in standstill. In contrast, the low-state of TT Ari is much lower and longer lasting, with few or no DN eruptions (carets denote upper limits). The data are 1-day averages based on compilations by the AAVSO, VSOLJ \& AFOEV.

turns into a ZC, and then into a NL. Thus VYs would have to be an intermediate stage similar to ZCs, perhaps between $\mathrm{ZC}$ and NL. However, the transition DN to ZC is marked by a trend to lower-amplitude, more frequent and more regular outbursts, with less time spent at minimum. The transition $\mathrm{ZC}$ to NL then sees periods of standstill increasing in frequency and length until they become all we see.

As remarked by Warner (1995), the VYs are distinctly different (see Fig. 3). The lightcurve changes have a greater amplitude (with an average $\Delta$ mag of 4.5 compared with 2.5 for ZCs; data from Ritter \& Kolb 1998); the low states are erratic and unpredictable; the star can spend months in a low state; such low states can have an $\dot{M}$ well below that of a $\mathrm{ZC}$ at minimum; the transitions between high and low states often take far longer than DN transitions, lasting up to $\sim 1 \mathrm{yr}$; and, in contrast to $\mathrm{ZCs}$, outbursts are rare or absent when in a low state. Further, if VYs are objects transitional in $\dot{M}$, similar to ZCs, why don't they occur at all periods above the gap, as NLs, ZCs and DN do? Why do VYs increase at $3<P<4 \mathrm{~h}$ whereas ZCs don't? And why is there no transitional object between a $\mathrm{ZC}$ and a VY?

From the dissimilarity of VY variability to DN and ZC variability, we conclude that we need a mechanism other than the disc instability to explain VYs. A big clue is that AM Her stars show low states very similar to those in VYs, and since they don't have discs the low states must involve changes in the mass transfered from the secondary star. Possibilities include the star-spot mechanism of Livio \& Pringle (1994), and irradiation-driven mass transfer cycles (Wu, Wickramasinghe \& Warner 1995). Irradiational heating of the secondary can increase the $\dot{M}$ above the rate justified by angular momentum loss, with the $\dot{M}$ leading to enhanced irradiation. This unstable feedback must break, plunging the system into a low state of near zero $\dot{M}$ as the secondary cools (see also Warner 1995 and Hellier 1996 for applications of this idea to VYs and SW Sex stars). The mechanism would require a high secular $\dot{M}$, and is more efficient in a close binary, producing the restriction of VYs to the period range just above the gap. In AM Hers the secondary is not shielded by the disc, allowing irradiation to be effective at lower $\dot{M}$ s.

If the above is correct, the deficit of $\mathrm{DN}$ with $3<P<4$ $\mathrm{h}$ would have to be explained by a different mechanism, or dismissed as chance. As discussed by Shafter (1992), a general increase in $\dot{M}$ could remove DN, but would have to be reconciled with theories of the width of the period gap.

\section{CONCLUSIONS}

This paper has been largely a defence of orthodoxy, showing that the period gap in cataclysmic variables is significant and that the nova-like variables show a cutoff at $\sim 3 \mathrm{hr}$. Further, we've argued against Verbunt's (1997) classification of VY Scl stars with the dwarf novae. Verbunt suggested that since VY Scl variability is probably caused by changes in mass transfer from the secondary star, this could also have a major role in DN outbursts. We've shown that the differences are sufficient to require separate mechanisms, such as the disc instability for DN and irradiation-driven mass transfer cycles for VY Scl stars.

\section{ACKNOWLEDGMENTS}

We thank Frank Verbunt for helpful comments on this work and gratefully acknowledge the contribution of amateur light-curve estimates complied by the AAVSO, VSOLJ and AFOEV. TN is a PPARC Advanced Fellow.

\section{REFERENCES}

Hellier C., 1996, ApJ, 471, 949

Hellier C., Mukai K., Beardmore A.P., 1997, MNRAS, 292, 397

Livio M., Pringle J. E., 1994, ApJ, 427, 956

Ritter H., Kolb U., 1998, A\&AS, in press

Shafter A. W., 1992, ApJ, 394, 268

Verbunt F., 1997, MNRAS, 290, L55

Warner B., 1995, Cataclysmic variable stars, Cambridge University Press, Cambridge

Warner B., 1996, Ap\&SS, 241, 263

Wickramasinghe D. T., Wu K., 1994, Ap\&SS, 211, 6

Wu K., Wickramasinghe D. T., Warner B., 1995, Publ. Astron. Soc. Aust., 12, 60 